\title{
Broadband Fivefold Reduction of Vacuum Fluctuations Probed by Dyes in Photonic Crystals
}

\author{
A. Femius Koenderink,* Lydia Bechger, H. P. Schriemer, Ad Lagendijk, and Willem L. Vos ${ }^{\dagger}$ \\ Van der Waals-Zeeman Instituut, Universiteit van Amsterdam, 1018 XE Amsterdam, The Netherlands
}

(Received 16 July 2001; published 20 March 2002)

\begin{abstract}
We observed for the first time a strong angle-independent modification of spontaneous emission spectra from laser dyes in photonic crystals, made of inverse opals in titania. Comparison with spectra from such crystals with much smaller lattice spacing, for which emission is in the long wavelength limit, reveals inhibition of emission up to a factor $\sim 5$ over a large bandwidth of $13 \%$ of the first order Bragg resonance frequency. The center frequency and bandwidth of the inhibition agree with calculated total density of states, while the measured inhibition of vacuum fluctuations is much larger. Because of the specific location of the dye molecules, we likely probe the strongly modulated local photonic density of states.
\end{abstract}

DOI: $10.1103 /$ PhysRevLett.88.143903

Within the framework of quantum electrodynamics (QED) it has long been recognized that the properties of the electromagnetic mode density may be used to tailor the fundamental atom-radiation interactions [1]. Numerous cavity QED experiments have demonstrated the effects of manipulating vacuum fluctuations. Prime examples are inhibition and enhancement of spontaneous emission and energy transfer, cavity induced energy level shifts, and van der Waals and Casimir forces [1]. Cavity QED experiments are generally limited to narrow spectral bandwidths, and small spatial volumes, i.e., near the center of the cavity. It is highly desirable to manipulate vacuum fluctuations over large bandwidths and extended spatial volumes in solid state systems, as proposed for so-called photonic band gap materials by Yablonovitch [2]. In this Letter, we report broadband inhibition of spontaneous emission in photonic crystals. This work opens up new research opportunities for studies of quantum optical phenomena in condensed matter environments.

Photonic crystals are periodic dielectric structures, with lattice spacings of the order of optical wavelengths [3]. Bragg diffraction causes the photonic dispersion to organize into bands, much like the energy levels of electrons in semiconductors. Important advances have been made with defect cavities in 1D [4] and in 2D photonic crystal slabs [5]. Complete suppression of vacuum fluctuations is expected in the case of a photonic band gap, i.e., a frequency range for which there are no wave vectors of propagation for any polarization. Such inhibition is hard to realize [6]. In pioneering experiments [7] on 3D photonic crystals, the inhibition was limited to a few percent by the small dielectric contrast [8].

Recently, novel routes have been reported to fabricate strongly interacting photonic crystals with high dielectric contrasts $[9,10]$. It has been experimentally shown [11] that the propagation of light is confined to less than $45 \%$ of all available directions at the high frequency edge of the lowest order Bragg diffraction, or $L$ gap. Hence, a major modification of the density of states (DOS) is expected. We examine the angle-independent spontaneous emission power of laser dyes inside such crystals, separated from
PACS numbers: 42.70.Qs, 42.25.Fx, 42.50.Ct, 81.05.Zx

the angle-dependent features reported earlier [12]. Reference spectra were obtained from chemically identical crystals with a much smaller lattice constant, for which the dye emission is in the long wavelength limit. In this limit the DOS is quadratic in frequency, as in a homogeneous medium [13-15]. The total emission power from the photonic crystals is clearly reduced for frequencies starting at the blue edge of the $L$ gap. The modification is observed to be independent of the choice of dye and occurs in a bandwidth of $13 \%$ of the $L$-gap center frequency, in agreement with the calculated modulation in the total DOS. The inhibition by a factor of $\sim 5$ reported here is much larger than the calculated change in the total DOS, which is the unit cell average of the local DOS (LDOS) [14-16]. Because of its specific spatial distribution, the dye probes the LDOS in part of the unit cell, near high index material. Although the spectral features are similar near the first order pseudogap, the LDOS is much more strongly modulated as a function of frequency than the total DOS $[14,16]$. Hence large changes in emission are expected.

We studied fcc crystals of air spheres in titania $\left(\mathrm{TiO}_{2}\right)$, with lattice parameters $a$ of 430,480, 510, and $350 \mathrm{~nm}$, respectively, the latter serving as a reference. As shown in Fig. 1 such inverse opals are strongly opalescent, have overall dimensions on the order of millimeters and are composed of high quality domains of dimensions up to $50 \mu \mathrm{m}$; for a detailed characterization, see Ref. [10]. Organic dyes were chosen as luminescent species for their small homogeneous [17] and large inhomogeneous linewidths, which allows one to probe the DOS in a large frequency range [18]. To adsorb the dye on the titania-air interfaces of the voids, air-sphere crystals with $a=350$, 430 , and $480 \mathrm{~nm}$ were immersed in a dilute $\sim 1 \mu \mathrm{mol} / 1$ solution of Rhodamine 6G (R6G) [19] in ethanol for $30 \mathrm{~min}$ and then rinsed and dried. Crystals with $a=350$ and $510 \mathrm{~nm}$ were similarly immersed for $24 \mathrm{~h}$, rinsed, and dried using a $70 \mu \mathrm{mol} / 1$ solution of Nile blue [19] in ethanol. To select emission from the bulk of the crystal, the dye adsorbed near the external sample surface was bleached by illuminating the crystals with an intense laser beam at the Bragg angle; see Ref. [18]. The dye 

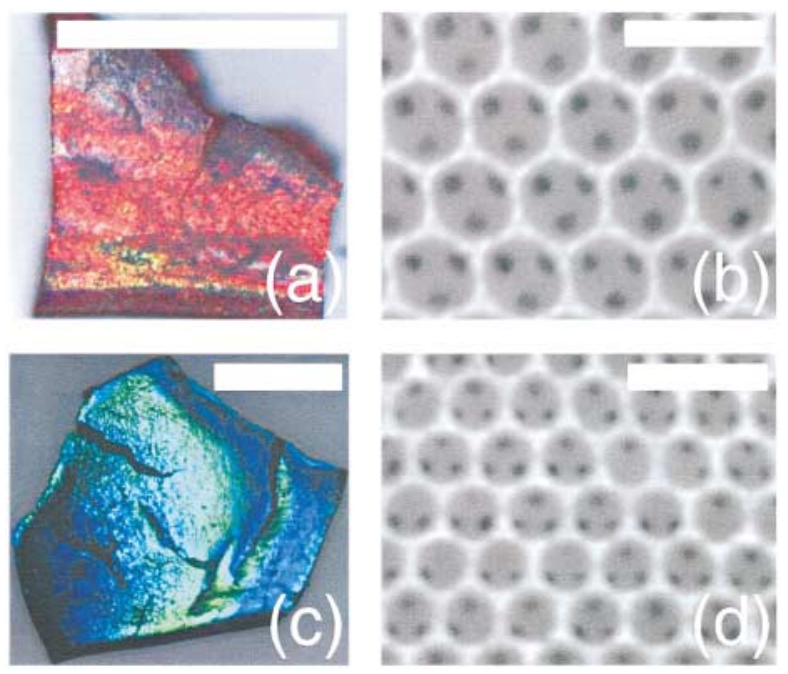

FIG. 1 (color). Optical micrographs (a) and (c) and scanning electron micrographs (b) and (d) of titania inverse opals with lattice constants $a=480 \mathrm{~nm}$, respectively, $a=350 \mathrm{~nm}$. Scale bars represent $0.5 \mathrm{~mm}$ in (a) and (c) and $500 \mathrm{~nm}$ in (b) and (d). Samples were illuminated with white light in (a) and (c).

molecules are located within a specific subset of less than $12 \%$ of the unit cell, comprising the solid material on which they are adsorbed [10].

The dye in the crystals was excited with a $p$ polarized laser beam, $\lambda=488 \mathrm{~nm}$ in the case of R6G and $\lambda=532 \mathrm{~nm}$ in the case of Nile blue, incident at angles away from the Bragg condition. Reference [12] provides a complete description of the setup used to detect $p$ polarized emission as a function of angle $\alpha$ relative to the 111 reciprocal lattice vector. For time resolved measurements, a mode-locked frequency-doubled Nd:YAG laser emitting 3 ps pulses at $12.5 \mathrm{~ns}$ intervals was used, in conjunction with a time-correlated single-photon counting technique providing a resolution of $55 \mathrm{ps}$.

In emission experiments designed to probe changes in the DOS, the efficiency of the luminescent source is of utmost importance. It is beneficial in a $\mathrm{cw}$ experiment to use a source with a low quantum efficiency: at a stationary pump rate $W$, the population of the excited state of the radiating species with total decay rate $\gamma_{\text {tot }}$ equals $W / \gamma_{\text {tot }}$, resulting in a photon emission rate $W\left(\gamma_{\mathrm{rad}} / \gamma_{\mathrm{tot}}\right)$. In the case of low quantum efficiency the emitted power is directly proportional to the radiative decay rate $\gamma_{\mathrm{rad}}$ and hence to the LDOS [16], since $\gamma_{\text {tot }}$ is dominated by nonradiative decay, and nearly independent of $\gamma_{\mathrm{rad}}$. In the case of high quantum efficiency $\left(\gamma_{\mathrm{rad}} / \gamma_{\mathrm{tot}} \rightarrow 1\right)$, changes in $\gamma_{\text {rad }}$ can be determined only in a time resolved measurement, since the emitted power will equal the pump rate. It is well known that organic dyes adsorbed on semiconductors have a low quantum efficiency, due to an electron transfer mechanism [20]. From a time resolved measurement, we conclude the quantum efficiency of R6G to be less than $8 \%$ of the $90 \%$ efficiency in ethanol [19]. Nile blue has a low efficiency $\leqslant 15 \%$ both in solution [19] and on titania. Therefore we have designed a $\mathrm{cw}$ experiment to probe the DOS.

Emission spectra at several detection angles for a R6G doped crystal with $a=480 \mathrm{~nm}$ (R6G) are presented in Fig. 2. Spectra are suppressed due to Bragg diffraction in a frequency range that shifts to higher frequency with increasing angle according to the band structure [12]. Modification of the spectra ceased for angles $\alpha$ exceeding $60^{\circ}$. The line shapes were independent of pump intensity, and the emitted intensity was linear with pump power. The spectral line shapes reproduced well for many spots on the sample surface, attesting to the crystal quality. Similar angle-dependent modification of the spectra was observed for samples with $a=430$ and $510 \mathrm{~nm}$, though shifted according to the lattice spacing. The $\alpha=60^{\circ}$ spectra are representative for the angle-averaged total emission power [21].

The $\alpha=60^{\circ}$ spectra for R6G doped samples with $a=$ $430,480 \mathrm{~nm}$ differ significantly from spectra of R6G in reference samples with $a=350 \mathrm{~nm}$, as shown in Fig. 3(a). A sizable reduction of emission power in the frequency window from $\sim 15500$ to $20000 \mathrm{~cm}^{-1}$ is apparent when the spectra are scaled to match on the low frequency side below any Bragg condition, resulting in clear redshifts of the maximum emission frequencies. A similar spectral shift was observed when comparing $\alpha=60^{\circ}$ spectra of the Nile blue sample $(a=510 \mathrm{~nm})$ with its reference. The line shapes reproduced well on a multitude of reference samples and were independent of angle, due to the fact that the lowest order Bragg diffraction occurs at $22000 \mathrm{~cm}^{-1}$ [see Fig. 1(c)]. The reduction is not due to differences in chemical interactions of the dye with its environment, since the reference samples are chemically identical to the photonic samples. Redshifts due to self-absorption are excluded, since the dye concentrations were sufficiently low by at least 2 orders of magnitude.

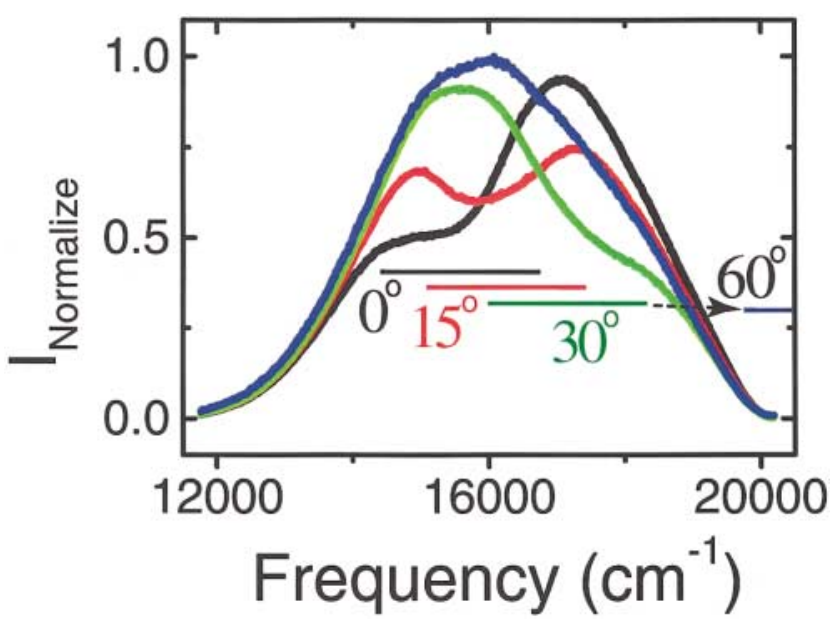

FIG. 2 (color). Emission spectra of R6G in a titania inverse opal with $a=480 \mathrm{~nm}$, obtained at angles $\alpha=0^{\circ}$ (black), $15^{\circ}$ (red), $30^{\circ}$ (green), and $60^{\circ}$ (blue). The attenuation range (colored bars) shifts to higher frequencies with angle, beyond the emission spectrum for $\alpha \geq 60^{\circ}$. 


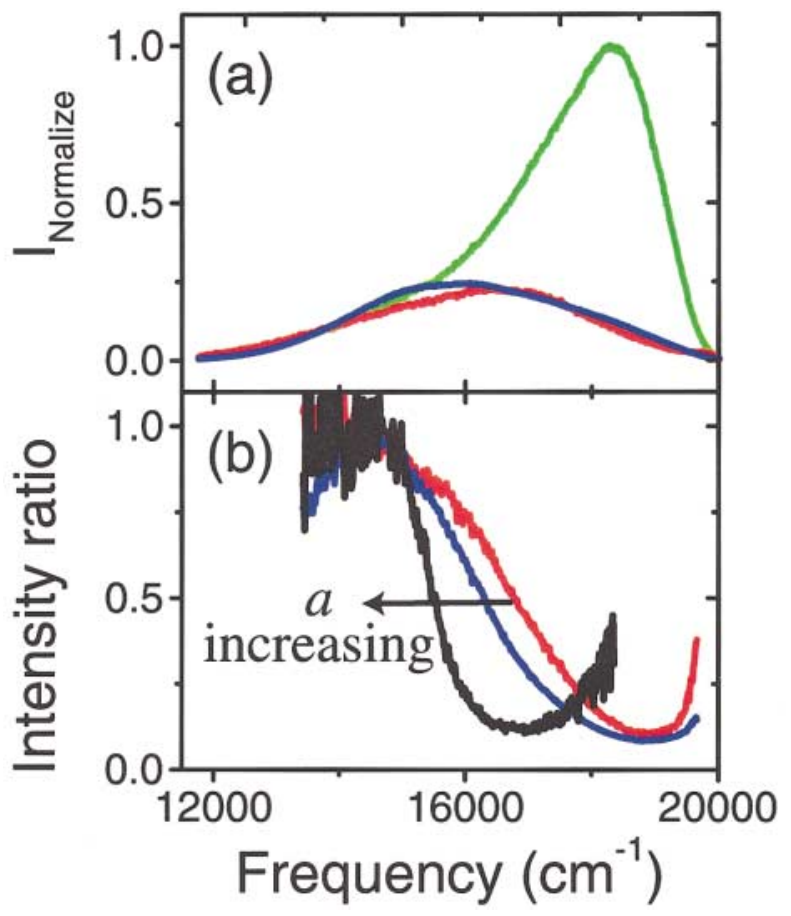

FIG. 3 (color). (a) Normalized emission spectra of R6G in titania inverse opals with $a=480 \mathrm{~nm}$ (blue), $a=430 \mathrm{~nm}$ (red) at $\alpha=60^{\circ}$, and reference crystal with $a=350 \mathrm{~nm}$ (green). (b) Intensity ratios of spectra in (a) to the reference spectrum for $a=480 \mathrm{~nm}$ (blue) and $a=430 \mathrm{~nm}$ (red). The black curve is the ratio of the $\alpha=60^{\circ}$ emission spectrum of Nile blue in a sample with $a=510 \mathrm{~nm}$ to the Nile blue reference spectrum. The inhibition shifts to the red with increasing lattice constant $a$.

We extract the photonic LDOS effect on the total emission by dividing the $\alpha=60^{\circ}$ spectra by the emission spectrum of the same dye in the reference samples, as shown in Fig. 3(b) for the Nile blue sample with $a=510 \mathrm{~nm}$ and the R6G data sets with $a=480,430 \mathrm{~nm}$. Because of the low efficiency of these dyes on titania, the intensity ratios, which are scaled to unity on the low frequency shoulder, represent the LDOS probed by the dye, divided by the reference LDOS. The lowest order stop gap of the reference samples at $22000 \mathrm{~cm}^{-1}$ is far above the dye spectrum. Hence, the reference LDOS has a well defined frequency dependence $[14,15]$, in contrast to a powder or pulverized sample of the same material which is strongly multiple scattering [22]. The width of the inhibition range $\sim 2000 \mathrm{~cm}^{-1}$, i.e., $13 \%$ of the $L$-gap center frequency, attests to the strongly photonic character of the inverse opals, which is robust to disorder [23]. The shift of the inhibition to higher frequency with smaller lattice parameter, confirms that the spectral changes are due to the photonic crystal properties.

It appears that the $\alpha=60^{\circ}$ spectra are representative of the spectral distribution of angle-averaged luminescence in the bulk, due to disorder effects. Since the transport mean-free path $\sim 15 \mu \mathrm{m}$ [24] is much smaller than the sample thickness $\sim 200 \mu \mathrm{m}$, the luminescence propagates diffusively through the bulk of the crystals. The spectral distribution of the diffuse luminescence is the $4 \pi$ sr average of spectra of all sources in the pump volume and is thus determined only by the LDOS and the inhomogeneous line broadening of the dye. The diffuse luminescence acquires an angular dependence (cf. Fig. 2) only when it exits the sample. This angle and frequency dependent modification of the diffuse luminescence spectrum is due to internal Bragg diffraction [12] and may be calculated from diffusion theory with internal reflection [25]. Estimating the internal reflectivity to be less than $70 \%$ for directions within a stop gap [12], and $0 \%$ outside, we find that the extraction efficiency for $\alpha=60^{\circ}$ increases by less than $30 \%$ as the frequency increases from the red to the blue edge of the $L$ gap, and linearly decreases by less than $10 \%$ as the frequency is increased to the blue edge of the emission spectra. Consequently, the $\alpha=60^{\circ}$ spectrum is representative of the diffuse luminescence spectrum in the bulk. Therefore the measured inhibition in Fig. 3 is due to the photonic DOS. Detailed analysis shows that the observed inhibition of emission in Fig. 3 is an upper bound to the true inhibition of total emission, due to a slightly enhanced extraction efficiency for $\alpha=60^{\circ}$ for frequencies in the inhibition band.

For comparison with the $\mathrm{cw}$ experiment, we have performed time resolved measurements on Nile blue, shown in Fig. 4. No significant difference is apparent between total decay rates in the photonic crystal and the reference, for frequencies either below or in the range of inhibited radiative decay evident from the spectra. The low quantum efficiency precludes determination of the radiative decay rate from a dynamic measurement but allows one to extract changes in radiative decay rate from the emitted power.

Remarkably, the magnitude of the reduction in emitted power, amounting to at least a factor of 5 relative to the low frequency edge, exceeds the reduction in total solid

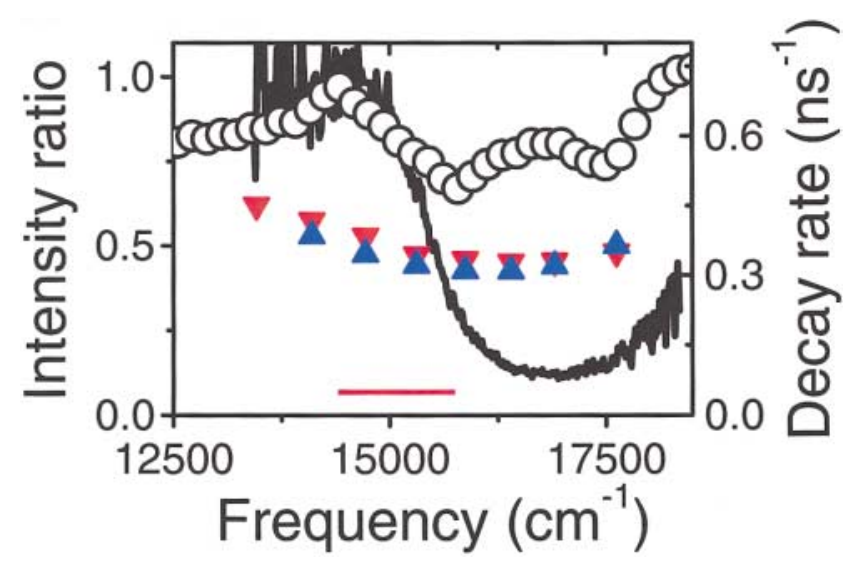

FIG. 4 (color). Total decay rates for Nile blue in the reference sample with $a=350 \mathrm{~nm}$ (blue) and photonic sample with $a=510 \mathrm{~nm}$ (red) with the scale indicated on the right axis. For comparison the $a=510 \mathrm{~nm}$ intensity ratio is shown (black curve). The open circles indicate the total DOS calculated for a titania inverse opal $a=510 \mathrm{~nm}$, divided by $\omega^{2}$ and scaled to match on the low frequency edge. The red bar indicates the calculated $L$ gap. 
angle [11] for light propagation by a factor of 2 , which is a simple estimate of the reduction of the total DOS. To investigate this further we have calculated the total DOS using the $H$-field inverted matrix technique [13,15]. For integration over the irreducible part of the fcc Brillouin zone we used an interpolative special point scheme [26], in which eigenfrequencies were calculated on a coarse grid of $2540 k$-points using 720 plane waves [27]. We used a dielectric model consisting of shells of titania $(\epsilon=6.5)$ with inner radius $R=a /(2 \sqrt{2})$, outer radius $1.09 R$, and cylindrical windows of radius $0.4 R$ connecting neighboring shells, based on structural data (Fig. 1 and Ref. [10]). This model predicts reflectivity features in excellent agreement with prior experiments [28]. As shown for the same model in Fig. 4, the total DOS normalized by the quadratic frequency behavior is reduced in a frequency range coincident with the experimentally determined inhibition window. Clearly, a complete understanding of the large reduction of the emitted power requires a full calculation of the LDOS, taking the spatial distribution and dipole orientation of the emitters into account. A time resolved decay measurement for efficient fluorescent sources would not show a simple single exponential decay but is composed of contributions from sources with different decay times depending on the LDOS at their positions.

In conclusion, we have observed for the first time broadband inhibition of spontaneous emission in a large spatial volume limited only by the size of the photonic crystal. This is a major step towards realizing novel quantum electrodynamics in controlled 3D defect cavities that are shielded from vacuum fluctuations. It is expected that pronounced maxima in the DOS of photonic crystals will be useful in applications requiring enhanced spontaneous emission in large spatial volumes [1,3].

This work is part of the research program of the "Stichting voor Fundamenteel Onderzoek der Materie (FOM)," which is financially supported by the "Nederlandse Organisatie voor Wetenschappelijk Onderzoek (NWO)."

*Email address: a.f.koenderink@utwente.nl

†Email address: w.l.vos@utwente.nl

${ }^{\ddagger}$ As of March 2002: Department of Applied Physics and $\mathrm{MESA}^{+}$Research Institute, University of Twente, 7500 AE, Enschede, The Netherlands.

URL: www.photonicbandgaps.com

[1] See, e.g., S. Haroche and D. Kleppner, Phys. Today 42, No. 1, 24 (1989); Y. Yamamoto and R. E. Slusher ibid. 46, No. 6, 66 (1993); M. O. Scully and M. S. Zubairy, Quantum Optics (Cambridge University Press, Cambridge, 1997); P. Andrew and W. L. Barnes, Science 290, 785 (2000).

[2] E. Yablonovitch, Phys. Rev. Lett. 58, 2059 (1987).

[3] Photonic Crystals and Light Localization in the 21st Century, edited by C. M. Soukoulis (Kluwer, Dordrecht, 2002).
[4] J. M. Gérard, B. Sermage, B. Gayral, B. Legrand, E. Costard, and V. Thierry-Mieg, Phys. Rev. Lett. 81, 1110 (1998).

[5] O. Painter, R. K. Lee, A. Scherer, A. Yariv, J. D. O'Brien, P. D. Dapkus, and I. Kim, Science 284, 1819 (1999); C. J. M. Smith, H. Benisty, D. Labilloy, U. Oesterle, R. Houdre, T. F. Krauss, R. M. De la Rue, and C. Weisbuch, Electron. Lett. 35, 228 (1999).

[6] G. Bourdon, I. Robert, R. Adams, K. Nelep, I. Sagnes, J. M. Moison, and I. Abram, Appl. Phys. Lett. 77, 1345 (2000).

[7] J. Martorell and N. M. Lawandy, Phys. Rev. Lett. 65, 1877 (1990).

[8] Z. Y. Li and Z. Q. Zhang, Phys. Rev. B 63, 125106 (2001).

[9] D. J. Norris and Y. A. Vlasov, Adv. Mater. 13, 371 (2001), and references therein.

[10] J.E. G. J. Wijnhoven and W.L. Vos, Science 281, 802 (1998); J. E. G. J. Wijnhoven, L. Bechger, and W. L. Vos, Chem. Mater. 13, 4486 (2001).

[11] M. S. Thijssen, R. Sprik, J. E. G. J. Wijnhoven, M. Megens, T. Narayanan, A. Lagendijk, and W. L. Vos, Phys. Rev. Lett. 83, 2730 (1999).

[12] H. P. Schriemer, H. M. van Driel, A.F. Koenderink, and W. L. Vos, Phys. Rev. A 63, 011801(R) (2001).

[13] K.-M. Ho, C. T. Chan, and C. M. Soukoulis, Phys. Rev. Lett. 65, 3152 (1990); H. S. Sözüer, J. W. Haus, and R. Inguva, Phys. Rev. B 45, 13962 (1992).

[14] T. Suzuki and P. K. L. Yu, J. Opt. Soc. Am. B 12, 570 (1995).

[15] K. Busch and S. John, Phys. Rev. E 58, 3896 (1998); Z. Y. Li and Y. Xia, Phys. Rev. A 63, 043817 (2001).

[16] R. Sprik, B. A. van Tiggelen, and A. Lagendijk, Europhys. Lett. 35, 265 (1996).

[17] M. D. Barnes, W. B. Whitten, S. Arnold, and J. M. Ramsey, J. Chem. Phys. 97, 7842 (1992).

[18] M. Megens, J.E. G. J. Wijnhoven, A. Lagendijk, and W. L. Vos, Phys. Rev. A 59, 4727 (1999); J. Opt. Soc. Am. B 16, 1403 (1999).

[19] U. Brackmann, Laser Dyes (Lambda Physik, Göttingen, 1997), and references therein.

[20] B. O'Regan and M. Grätzel, Nature (London) 353, 737 (1991); P. V. Kamat, Chem. Rev. 93, 267 (1993).

[21] Because of fcc symmetry, experiments in the angular range $0^{\circ} \leq \alpha \leq 60^{\circ}$ effectively probe all of the $4 \pi$ solid angle.

[22] B. A. van Tiggelen and E. Kogan, Phys. Rev. A 49, 708 (1994).

[23] Z. Y. Li and Z. Q. Zhang, Phys. Rev. B 62, 1516 (2000).

[24] A. F. Koenderink, M. Megens, G. van Soest, W. L. Vos, and A. Lagendijk, Phys. Lett. A 268, 104 (2000).

[25] J. X. Zhu, D. J. Pine, and D. A. Weitz, Phys. Rev. A 44, 3948 (1991); D. J. Durian, Phys. Rev. E 50, 857 (1994).

[26] H. J. Monkhorst and J. D. Pack, Phys. Rev. B 13, 5188 (1976); G. Gilat, J. Comput. Phys. 10, 432 (1972).

[27] In this frequency range, the bands and total DOS are insensitive to a further increase of the number of plane waves or $k$ points. Calculations for fcc close packed air spheres in $\epsilon=11.9$ agree well with Busch and John [15], Fig. 8.

[28] H. M. van Driel and W.L. Vos, Phys. Rev. B 62, 9872 (2000); W. L. Vos and H. M. van Driel, Phys. Lett. A 272, 101 (2000). 